\title{
Pulmonary Artery Pseudoaneurysm with Recurrent Massive Haemoptysis: Endovascular Management by a Covered Stent
}

\author{
Naseer A. Choh ${ }^{1}$ Saika Amreen ${ }^{1, \odot ~ M u d a s i r ~ H a m i d ~}{ }^{1} \quad$ Imran Nazir ${ }^{1}$ \\ ${ }^{1}$ Department of Radiodiagnosis and Imaging, Sher-i-Kashmir \\ Institute of Medical Sciences, Srinagar, Jammu and Kashmir, India \\ Address for correspondence Saika Amreen, MD, Department of \\ Radiodiagnosis and Imaging, Sher-i-Kashmir Institute of Medical \\ Sciences, Soura, Srinagar, Jammu and Kashmir 190010, India \\ (e-mail: saikaamreen@gmail.com).
}

J Clin Interv Radiol ISVIR 2021;6:64-67.
Abstract
Keywords
- pulmonary artery pseudoaneurysm
- covered stent
- thrombin

Pulmonary artery pseudoaneurysm (PAPA) is a potentially fatal, albeit rare cause of hemoptysis. With a varied etiology and debatable management, time is the essence in its treatment. We present a case of a post lobectomy PAPA with recurrence of malignancy, managed via endovascular approach in a low resource setting where an ideal hardware was not available.

\section{Case Report}

A 65-year-old man who had undergone right upper lobectomy and completed chemoradiotherapy one year back for lung malignancy came with a history of massive haemoptysis. Contrast enhanced computed tomography revealed a large right pulmonary pseudoaneurysm arising from the proximal lower lobar artery, with a narrow neck ( - Fig. 1). There was pleural thickening and patchy consolidation of the lung and prior lobectomy changes. Adjacent to the pseudoaneurysm a soft tissue density lesion was observed which could be due to recurrence of malignancy.

The patient was referred to our Interventional Radiology service for management as a repeat thoracotomy could be associated with high risk of complications and mortality. A covered stent was thought to be the ideal approach, but the required size was not available. It was decided to employ balloon assisted thrombin injection in an attempt to thrombose the pseudoaneurysm. Right femoral access was obtained using a $6 \mathrm{~F}$ sheath. After taking a pulmonary angiogram, a microcatheter was introduced into the pseudoaneurysm followed by careful, complete balloon occlusion of the right lobar pulmonary artery using a $12 \mathrm{X} 40 \mathrm{~mm}$ balloon (Mustang non-complaint balloon from Boston) in an attempt to induce stasis. Low inflation pressure of 6-8 ATM was used. 1500U of thrombin was injected along with gel foam slurry and balloon was deflated after five minutes. The check angiogram revealed $80 \%$ thrombosis of the pseudoaneurysm (-Fig. 2).

The patient after a brief period of stabilization (4 days) again presented with bouts of massive haemoptysis and it was decided to place a covered stent in the right lower lobe pulmonary artery ( - Fig. 3). A $10 \mathrm{~F}$ and $6 \mathrm{~F}$ venous access was obtained, both via right femoral vein, utilizing $6 \mathrm{~F}$ for a pigtail catheter and $10 \mathrm{~F}$ for the covered stent. The $10 \mathrm{~F}$ access was used for deploying the stent, while the $6 \mathrm{~F}$ was used to take selective runs of the pulmonary artery during deployment for precise location proximally as well as distally. After confirmation of position, using an exchange length Amplatz extra stiff guidewire, a $14 \mathrm{~mm}^{*} 4 \mathrm{~cm}$ covered self-expandable covered stent (Bard Fluency Plus) was quickly deployed as the patient was bleeding massively ( - Fig. 4 ). No heparin was given during the procedure as the patient was bleeding profusely and the procedure was completed under 25 minutes with the patient in conscious sedation. The patient published online

June 1, 2021
DOI https://doi.org/

$10.1055 / \mathrm{s}-0041-1730105$

ISSN 2457-0214 (c) 2021. Indian Society of Vascular and Interventional Radiology.

This is an open access article published by Thieme under the terms of the Creative Commons Attribution-NonDerivative-NonCommercial-License, permitting copying and reproduction so long as the original work is given appropriate credit. Contents may not be used for commercial purposes, or adapted, remixed, transformed or built upon. (https://creativecommons.org/licenses/by-nc-nd/4.0/).

Thieme Medical and Scientific Publishers Pvt. Ltd. A-12, 2nd Floor, Sector 2, Noida-201301 UP, India 


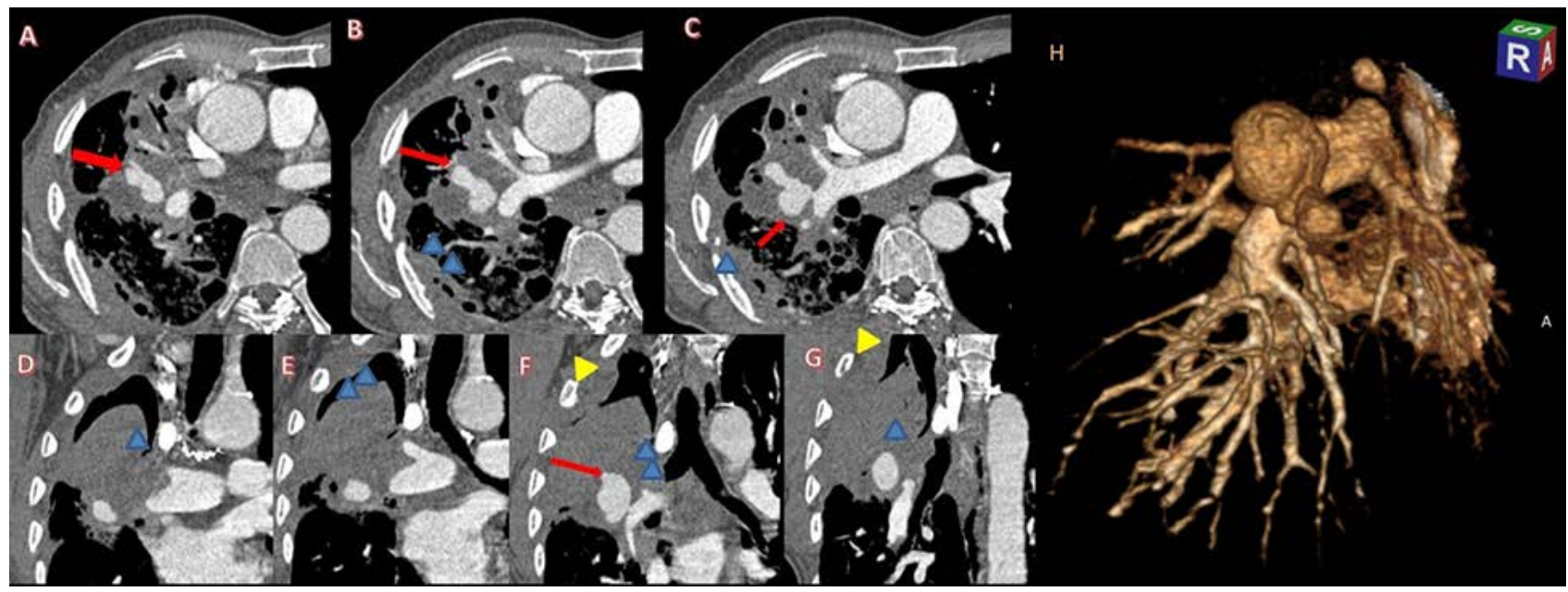

Fig. 1 Contrast enhanced computed tomography, Axial (A-C) and coronal (D-G) images depicting the large pseudoaneurysm (red arrows) and marked nodular pleural thickening(blue arrowheads) with rib erosion (yellow arrowhead) suggestive of recurrence. H: 3D VRT image depicting the large pseudoaneurysm.

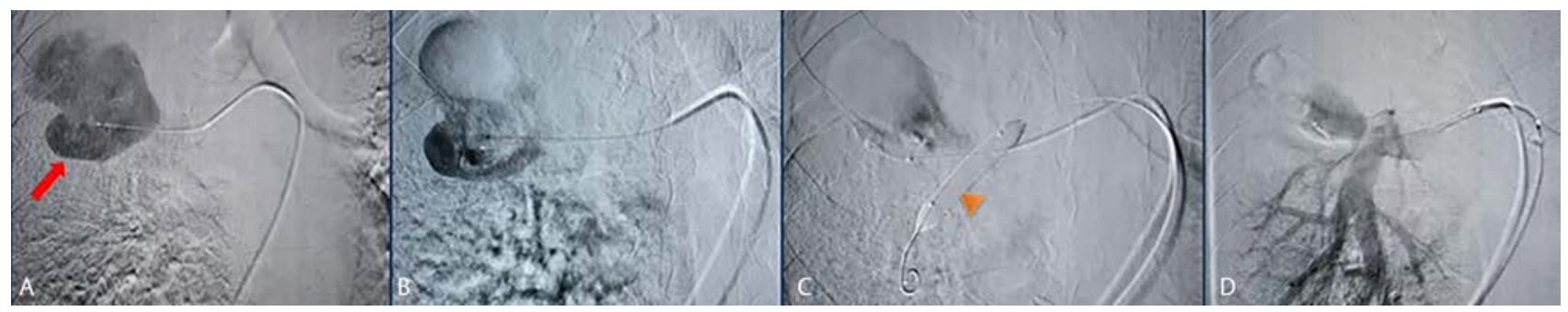

Fig. 2 Pulmonary angiogram depicting a microcatheter introduced into the pseudoaneurysm (red arrow) followed by careful balloon occlusion of the right lobar pulmonary artery (orange arrowhead) and thrombin injection (A-C) The check angiogram revealed $80 \%$ thrombosis of the pseudoaneurysm (D).

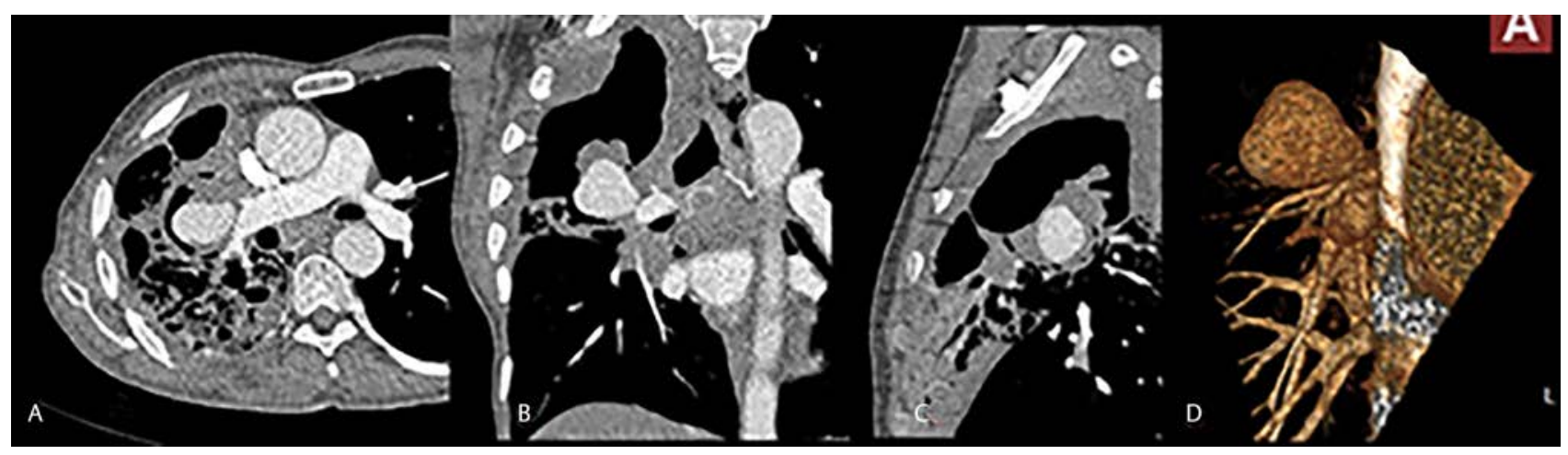

Fig. 3 A repeat contrast CT revealed refilling of the pseudoaneurysm (A-C: axial, coronal and sagittal images, D: 3D VRT image).

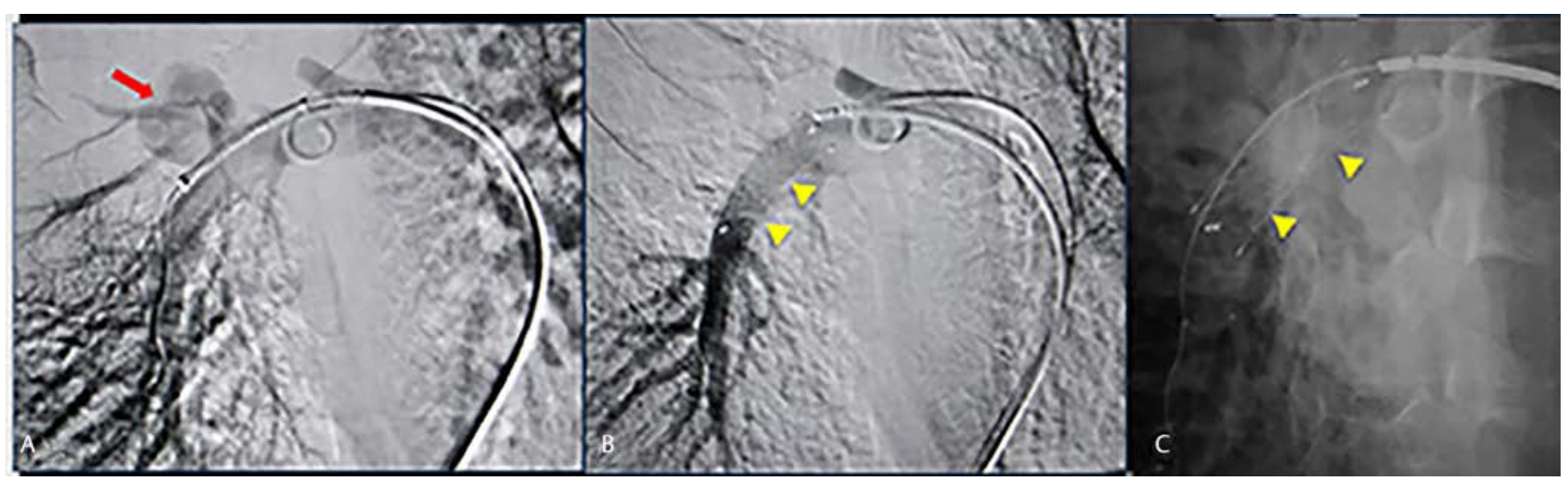

Fig. 4 Pulmonary angiogram depicting the pseudoaneurysm (red arrow). A covered stent (Bard) (yellow arrowhead) was deployed (B-C). 


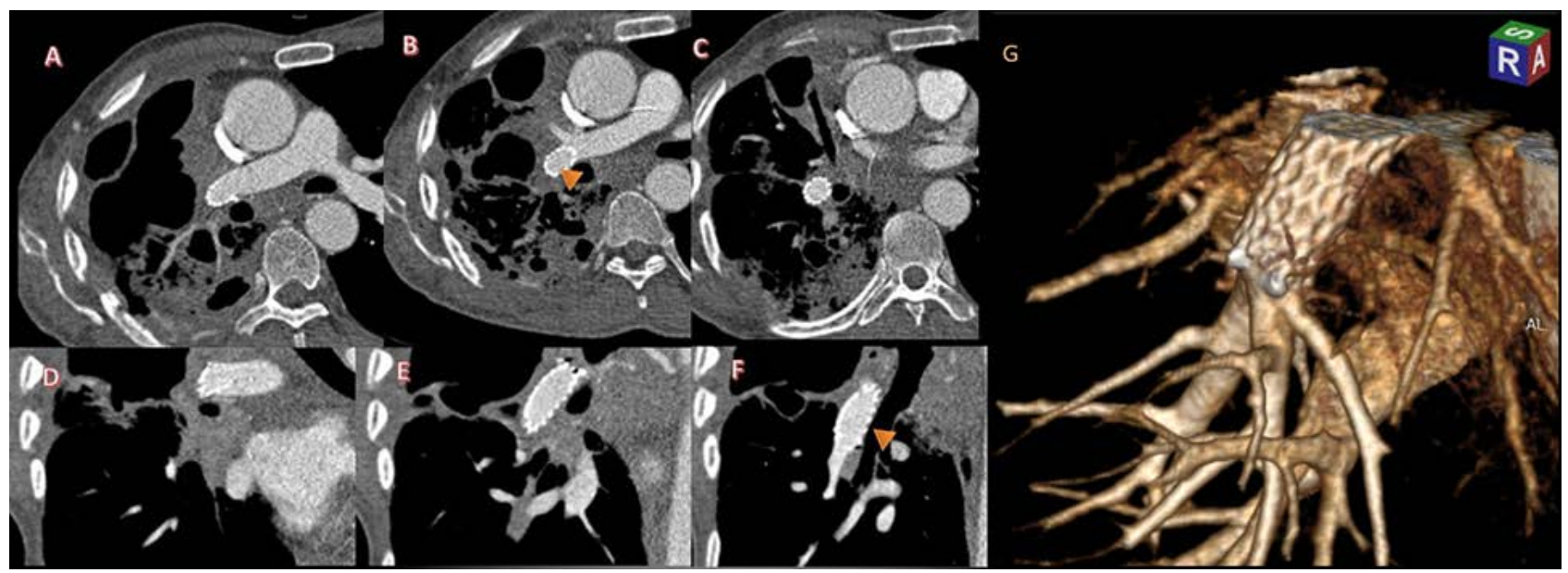

Fig. 5 Repeat contrast CT, Axial (A-C), coronal (D-F) and VRT (G) images from the check CT angiogram revealing complete thrombosis of the pseudoaneurysm with the stent in situ. (orange arrowhead).

stabilized but continued to have mild episodic haemoptysis for 24 hours. A check CT angiogram revealed complete thrombosis of the pseudoaneurysm with a small pulmonary infarct ( - Fig. 5). Patient was put on maintenance dose anticoagulation consisting of aspirin 75mg OD and clopidogrel 75mg OD. Clopidogrel was stopped after 3 months and aspirin $75 \mathrm{mg}$ was continued. Haemoptysis ceased and the patient lived for over a year, subsequently dying due to myocardial infarction.

\section{Discussion}

A pulmonary artery pseudoaneurysm is a rare, potentially fatal condition which can be caused by a multitude of factors including but certainly not limited to pulmonary resection, abscesses, collagen vascular disease, infection, trauma, iatrogenic cause etc. It warrants emergent surgery which may often be difficult and have a high chance of mortality. The utilization of endovascular treatment provides a new frontier in the management. ${ }^{1-5}$ PAPAs have been reported in association with lung cancer, attributed to direct invasion by the malignancy. ${ }^{3,6}$ Few have however been reported post lobectomy. ${ }^{1,7}$

Open surgical management can be argued to be the first choice in the treatment of a PAPA, especially in cases when it has associated abscess or a lesion that will benefit from resection. Options include pulmonary artery ligation, segmentectomy or lobectomy, arterioplasty or placement of a pericardial patch. However, cases with past surgical history where the operative field is likely to be ridden with adhesions, or in debilated and run down patients with an anticipated high morbidity and mortality, endovascular repair is a viable option. ${ }^{1,3-5}$ Coil embolization has also been used to treat the PAPA with the primary complications of pulmonary infarction..$^{1-3,8}$ Placement of a stent graft has also been reported in treatment of symptomatic PAPA, with the advantage of preservation of distal blood flow. ${ }^{4,5,9}$ Percutaneous thrombin injection has been used usually after inadequate treatment by coil embolization. ${ }^{10}$

\section{Conclusion}

Endovascular management is a safe and minimally invasive option for treatment of PAPA, especially in non surgical candidates. This case highlights the value of having a wide range of inventory of covered stents of different sizes for bleeding visceral pseudoaneurysms so that immediate treatment can be instituted. As this pseudoaneurysm was directly communicating with the proximal right pulmonary artery, neither coiling nor plug deployment was possible. The thrombin injection was not satisfactory as is true of pseudoaneurysms with rapid flow. It however, did stabilize the patient buying time till a covered stent was available.

\section{Conflict of Interest}

None declared.

\section{References}

1 Matsumura Y, Shiono S, Saito K, Sato T. Pulmonary artery pseudoaneurysm after lung resection successfully treated by coil embolization. Interact Cardiovasc Thorac Surg 2010;11(3):364-365

2 de Jonge I, Vahl A, van der Hulst V. Coil embolization of a left pulmonary artery pseudoaneurysm after penetrating injury. J Endovasc Ther 2003;10(3):681-683

3 Akpinar E, Turkbey B, Canyigit M, et al. Bleeding pulmonary artery pseudoaneurysm secondary to squamous cell lung cancer: computed tomography findings and endovascular management. Acta Radiol 2006;47(9):944-946

4 Park A, Cwikiel W. Endovascular treatment of a pulmonary artery pseudoaneurysm with a stent graft: report of two cases. Acta Radiol 2007;48(1):45-47

5 Huet N, Rodiere M, Badet M, et al. Covered Stent and Coils Embolization of a Pulmonary Artery Pseudoaneurysm 
After Gunshot Wound. Cardiovasc Intervent Radiol 2016; 39(5):778-781

6 Camargo Jde, Camargo SM, Machuca TN, Bello RM. Large pulmonary artery pseudoaneurysm due to lung carcinoma: pulmonary artery pseudoaneurysm. J Thorac Imaging 2010;25(1):W4-5

7 Shaaban H, Sharma H, Rao J, Clark S. A pulmonary artery false aneurysm after right middle lobectomy: a case report. J Med Case Reports 2007;1(1):70

8 Bao M, Zhou Y, Jiang G, Chen C. Pulmonary artery pseudoaneurysm after a left upper sleeve lobectomy. World J Surg Oncol 2013;11:272
9 Palmieri VJ, Heal ME, Chowdhury SM, Bandisode VM. Balloon-expandable covered stent implantation for treatment of a traumatic pulmonary artery pseudoaneurysm in a pediatric patient. J Thorac Cardiovasc Surg 2018;155(2):e73-e76

10 Hovis CL, Zeni PT Jr. Percutaneous thrombin injection of a pulmonary artery pseudoaneurysm refractory to coil embolization. J Vasc Interv Radiol 2006;17(12):1943-1946 